\title{
Hemorragia de tubo digestivo alto
}

\author{
Joel O. Jáquez-Quintana* \\ Servicio de Gastroenterología y Endoscopia Digestiva, Hospital Universitario José E. González, U.A.N.L, Monterrey, N.L., México
}

\section{Introducción}

La hemorragia digestiva alta (HDA) es un problema común que se estima que ocurre en 80 a 150 de cada 100,000 personas cada año. Las tasas de mortalidad estimadas están entre el 2 y el 15\% $\%^{1,2}$.

De las posibles etiologías de HDA, la enfermedad de úlcera péptica (EUP) representa del 40 al $50 \%$ de los casos. De ellos, la mayoría es secundaria a úlceras duodenales (30\%). La EUP se puede relacionar con antiinflamatorios no esteroideos (AINE), Helicobacter pylori y enfermedad de las mucosas relacionada con el estrés ${ }^{1}$.

Aparte de la EIP, la esofagitis erosiva representa el $11 \%$, la duodenitis el $10 \%$, las várices del 5 al $30 \%$ (dependiendo de si la población estudiada tiene una enfermedad hepática crónica), el desgarro de Mallory-Weiss el 5 al $15 \%$ y las malformaciones vasculares el $5 \%$. En este resumen se valorarán temas como tiempo de ingreso del paciente y endoscopia (endoscopia urgente vs. temprana), así como procedimientos endoscópicos en fin de semana, y si hay algún impacto en la morbilidad y mortalidad, así como efecto del manejo conservador y retraso de la endoscopia superior en el manejo de la HDA durante la pandemia en pacientes con COVID-19.

Los estudios presentados en la DDW uno de los más interesantes es el Dr. Masayasu Horibe, de la Universidad de Keiko, Tokyo (Japón) y la Clínica Mayo, el cual evalúo la influencia en la mortalidad a los 30 días de la hemostasia endoscópica urgente $(<6$ h) vs. temprana ( $>6 \mathrm{~h}$ ) en pacientes con sangrado de tubo digestivo alto con estigmas de alto riesgo (EAR). Evaluando de 1,966 pacientes de los cuales se incluyeron 886 pacientes con EAR, en donde a 769 pacientes se les realizó endoscopia urgente $(<6$ h) y 117 pacientes endoscopia temprana ( $>6 \mathrm{~h}$ ). Los resultados que obtuvo de los 886 pacientes con EAR en el 769 pacientes la endoscopia se realizó en las primeras 3 horas (rango intercuartil: 2-4 h) y en 117 pacientes a las 12 horas (rango intercuartil: 8.5-19 h) con una mortalidad global de 35/866 (4\%). En el análisis multivariante el éxito de la hemostasia endoscópica y la endoscopia urgente $(<6$ h) se asoció a una reducción de la mortalidad, (odds ratio [OR]: 0.24; IC 95\%: 0.11-0.58; $p=0.0025$ ) y (OR: 0.34; IC 95\%: 0.14-0.99; $p=0.047$ ) respectivamente. Sin embargo, se conformaron 2 grupos de 115 a los cuales aplico la escala de Harbinger ${ }^{3}$ (que consta de 3 variables: ausencia de uso diario de inhibidores de la bomba de protones [IBP] en la semana anterior a la presentación del sangrado digestivo; estado de choque [frecuencia cardiaca/presión arterial sistólica $\geq 1$ y urea en sangre nitrógeno/creatinina $\geq 30$ ) y con base en esta escala decidir endoscopia urgente 0 temprana, observando una mortalidad más baja en aquellos que se les realizó una endoscopia urgente vs. endoscopia temprana (2.61 vs. $7.83 \%$; $p<0.0001$ ). Concluyendo que los pacientes con sangrado digestivo alto y EAR se benefician de una endoscopia urgente y control hemostásico endoscópico para reducción de la mortalidad y que se puede utilizar la escala de Harbinger puede predecir la presencia de los EAR ${ }^{4}$.

\section{Correspondencia:}


Además, se presentó otro estudio del Dr. Andrés Rodríguez de la University of Miami/Jackson Memorial Hospital. En donde evaluó la intervención endoscópica temprana en hemorragia gastrointestinal superior basada en el día de admisión, retrospectivamente de una base de datos identifico pacientes con HDA variceal (HDA-V) y no variceal (HDA-NV), definiendo aquellos que se les realizó endoscopia temprana a los que se les realizó endoscopia superior en las primeras 24 horas posterior ingreso, así como determinado se fue esta en finde semana si el ingreso fue viernes media noche hasta domingo media noche. En los resultados se observó que la proporción de endoscopia urgente los fines de semana es mayor en aquellos paciente con HDA-V vs. HDA-NV $(p<0.001)$ y de la misma forma esto era mayor en fines de semanas que entre semana $(p<0.05)$. Y al compararse con un hospital de New York y Massachusetts fue la misma tendencia ${ }^{5}$. De tal forma, entonces, esto refleja que ciertos hospitales mantienen protocolos de manejo consistentes en salas de endoscopia, sin embargo, valdría la pena determinar la morbilidad y mortalidad relacionada al adecuado manejo médico de los pacientes, así como una endoscopia con control de la hemorragia, ya que no solo es hacer la endoscopia si no tener equipo médico, completo y capacitad o así como acceso a material y accesorios.

En cuanto a sangrado digestivo y el impacto de la pandemia de COVID-19 en el manejo y resultados clínicos de pacientes ingresados con sangrado gastrointestinal superior el Dr. Ekawat Manomaiwong del hospital Siriraj, Mahidol, Bangkok (Thailandia), valoró de forma retrospectiva de abril a junio 2020 el impacto del tratamiento conservativo en el desenlace de los pacientes con HDA en pacientes con COVID-19 comparando el mismo periodo del año previo a la pandemia. En cuanto resultados valoró 60 pacientes con HDA con COVID-19 vs. HDA sin COVID-19 460, en cuanto a características demográficas los pacientes con HDA con COVID-19 fueron de mayor edad $67+$ $14.25-62.75+15.52(p=0.045)$, en cuanto sexo, índice de masa corporal, enfermedad cardiaca-renal, hepática fueron equiparables, no así la presencia de tumores solidos con 30 vs. $18(p=0.028)$ respectivamente, HDA con COVID-19 vs. HDA sin COVID-19. EN cuanto al procedimiento de endoscopia superior (EDS), esta se realizó en HDA con COVID-19 en el $43.3 \%$ vs. HDA sin COVID-19 en el 95\% ( $p<0.001)$, y en los que se realizó la EDS, esta fue en las primeras $24 \mathrm{~h}$ en $6.7 \%$ vs. $45.2 \%(p<0.001)$ respectivamente con una media en el tiempo de la endoscopia en horas en pacientes con HDA con COVID-19 de 70 h (intervalo 48-111 h) vs. HDA sin COVID-19 25 h (intervalo de 16-48 h). En cuanto impacto en desenlace y complicaciones, no se documentaron diferencias en resangrado a 30 días, sin embargo, si hubo más infecciones (23.3 vs. $12.6 \%$; OR: 2.109; IC 95\%: 1.092-4.075; $p=0.024$ ) en aquellos con HDA con COVID, además de mayor mortalidad a 30 días (13.3 vs. $6.1 \%$; OR: 2.1374; IC 95\%: 1.028$5.480 ; p=0.043)$, por otro lado, al analizar el número de transfusión de paquete globulares 3 (1-6) vs. 2 (1-3) ( $p=0.002$ ), y aumento en días de estancia hospitalaria, 5 días (rango: 4-12) vs. 4 días (rango: 2-6) $(p=0.002)$ en el mismo grupo de pacientes de HDA con COVID. y un subanálisis de aquellos con HDA con COVID a los cuales no se les realizó EDS vs. a los que si se realizó EDS y comparando la EDS prepandemia era mayor la mortalidad en los que no se realizó EDS, solo que no se determinó causa de muerte si fue por complicaciones del COVID-19 o del STDA ${ }^{6}$. Este estudio demuestra el actuar de forma conservadora ante el HDA durante la pandemia del SARS-CoV-2 si tuvo un impacto en pacientes con HDA. Por otro lado sería interesante analizar aquellos pacientes con HDA a los cuales se retrasó o no se realizó la EDS, cual fue la presentación clínica, si el HDA fue la causa del ingreso hospitalario o si el HDA se presentó durante el manejo del COVID-19 y su relación con gravedad. Estos son datos recientes al inicio de la pandemia, así como reportes donde tanto la incidencia de HDA con y sin COVID es la misma, al igual que presentación clínica ${ }^{7}$. Finalmente, el escenario de HDA y COVID es nuevo y de forma gradual se fue conociendo la enfermedad y estableciendo protocolos de manejo, lo cual debe de seguirse analizando y contestar muchas preguntas a las cuales nos enfrentamos durante esta pandemia.

\section{Financiamiento}

La presente investigación no ha recibido ayudas específicas provenientes de agencias del sector público, sector comercial o entidades sin ánimo de lucro.

\section{Conflicto de intereses}

Los autores declaran no tener conflicto de intereses.

\section{Bibliografía}

1. Kamboj AK, Hoversten $P$, Leggett $C L$. Upper gastrointestinal bleeding: Etiologies and management. Mayo Clin Proc. 2019;94:697-703. 
Endoscopia. 2021;33(Supl 1)

2. Stanley AJ, Laine L. Management of acute upper gastrointestinal bleeding. BMJ. 2019;364:1536.

3. Horibe M, Iwasaki E, Bazerbachi F, Kaneko T, Matsuzaki J, Minami K, et al. Horibe Gl bleeding prediction score: a simple score for triage decision-making in patients with suspected upper Gl bleeding. Gastrointest Endosc. 2020;92:578-88

4. Horibe M, et al. Superiority of urgent vs. early endoscopic hemostasis in patients with upper gastrointestinal bleeding. Gastroenterology. Año;160(6):S-655
5. Rodriguez AD, et al. Su259 Evaluation of early endoscopic intervention with upper gastrointestinal hemorrhages based on day of admission. Gastroenterology. Año;160(6):S-659.

6. Manomaiwong $E$, et al. Sa110 Impact of COVID-19 pandemic on the management and clinical outcomes of patients admitted with upper gastrointestinal bleeding. Gastroenterology. Año;160(6):S-424.

7. Garcia-Sague, B et al. Non variceal upper gastrointestinal bleeding during Covid 19 outbreak. incidence and clinical characteristics. Endoscopy. 2021;53(S01):264 